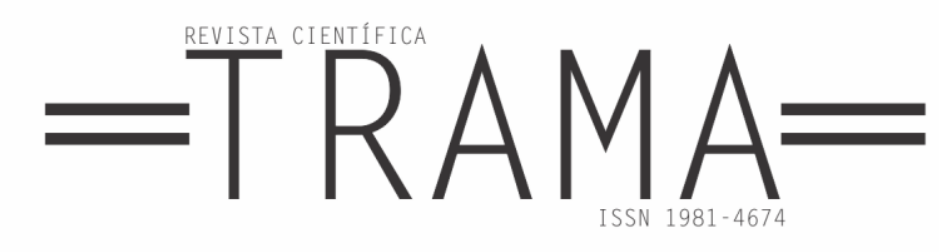

\title{
W IE diE SCHWEIZER KOLONIE HELVETIA IN SÃO PAULO VON OBWALDNERTYYTSCH ZU PORTUGIESISCH WECHSELTE
}

\author{
Andreia Caroline KARNOPP1
}

\begin{abstract}
Zusammenfassung: In diesem Artikel soll überblickshaft beschrieben werden, wie sich die Kontaktsprachen in der Schweizer Kolonie Helvetia im Staate São Paulo entwickelt haben. Dazu wird zunächst erläutert, wie es Schweizer geschafft haben, eine Sprachinsel in Brasilien aufzubauen und bis heute zu erhalten. Dann wird der Sprachwechsel mittels der Schulgeschichte Helvetias aufgezeigt und der aktuelle Vitalitätsstatus des Dialekts und die noch genutzten Sprachdomänen beschrieben. Abschliessend werden einige konkrete Sprachbeispiele dargelegt.
\end{abstract}

Schlüsselwörter: Sprachinsel, Sprachwechsel, Sprachkontakt.

\begin{abstract}
Resumo: No presente artigo pretende-se dar uma visão geral como as línguas em contato evoluiram na colônia suiça $\mathrm{Hel}$ vetia no estado de São Paulo. Com este propósito explica-se primeiramente, como os suíços conseguiram formar uma enclave linguística no Brasil e como eles conseguiram mantê-la. No que segue descreve-se com a história escolar de Helvetia a mudança de idioma. Também serão ilustrados o estado de vitalidade do dialeto suíço e os seus atuais domínios linguísticos. Para concluir seguem alguns exemplos concretos.
\end{abstract}

Palavras-chave: Enclave linguística, mudança de língua, contato linguístico.

Recebido em 31-08-2017 Aceito em 24-10-2017

\footnotetext{
${ }^{1}$ Doutoranda em Linguística Portuguesa sobre "o português dos helvetianos em São Paulo", no departamento de Línguas Românicas na Universidade de Zurique (Suíça), sob coordenação de Prof. Dr. Johannes Kabatek e de Prof. Dr. Stephan Schmid.
} 


\section{$=$ TRAMA $=$}

E

\section{ntstehung der kolonie in São Paulo}

Vor rund $160 \mathrm{Jahren}^{2}$ sind die ersten Familien aus dem Schweizer Kanton Obwalden nach Brasilien ausgewandert. Grund dafür war u.a. die Kraut- und Kartoffelfäulnis, die in den 1840er Jahren in ganz Europa eine große Hungersnot verursachte. In diesem Zusammenhang verarmten viele Bauern und andere Unterschichtsangehörige (u.a. Historisches Lexikon der Schweiz online, Kartoffel). Eine willkommene Lösung war deswegen die organisierte Auswanderung ${ }^{3}$ in ein neues Land, wo den Betroffenen ein besseres Leben versprochen wurde (u.a. STREIFF-BECKER, 1957). Was diese dort jedoch vorfanden waren nicht nur völlig andere Lebensumstände und schlechtbezahlte Anstellungen in Kaffeeplantagen, sondern auch das Problem der Sprache.

Den ersten Auswanderern war es unmöglich, ihre Herkunftssprache (also das Ur-Obwaldnertyytsch, einen Schweizerdeutschen Dialekt aus dem Kanton Obwalden, der im 19. Jahrhundert gesprochen wurde ( $c f$. IMFELD, 2001), beizubehalten. Einerseits gab es zu diesem Zeitpunkt keine Schweizer Schule, andererseits waren sie außerhalb ihrer eigenen vier Wände ständig mit der neuen Landesprache Portugiesisch konfrontiert - welche sie übrigens von den bereits auf den Kaffeeplantagen arbeitenden afrikanischen Sklaven lernten (WEIZINGER, 2005).

Um 1880 reisten weitere Obwaldner Familien nach São Paulo, nur

\footnotetext{
${ }^{2}$ Die ersten Schweizer waren bereits im Jahr 1816 (bzw. 1824 - letzteres Datum wird als offizielles Einwanderungsdatum der deutschsprachigen Bevölkerungsgruppen angesehen) nach Übersee ausgewandert. Die erste offizielle Deutsch-Schweizer Kolonie auf brasilianischem Grund ist die 1816 gegründete Kolonie Leopoldina (heute Helvécia), und liegt in Nova Viçosa im Staat Bahia.
}

war dieses Mal die Situation eine andere: Die Reise war freiwillig, ging nicht mehr ins Unbekannte und zudem stand Brasilien kurz davor, die Sklaverei abzuschaffen (WEIZINGER, 2005). Aus diesem Grund suchten die Großgrundbesitzer verzweifelt neue Arbeitskräfte. Die frisch eingereisten Innerschweizer kamen bereits mit etwas Erspartem ins neue Heimatland und konnten durch die gutbezahlte Arbeit auf der Fazenda Sitio Grande (heute Fazenda São Luís) in Itupeva/Jundiaí zusätzlich Geld ansparen. Am 14. April 1888 haben sich mehrere Schweizer Familien zusammengetan (überliefert sind die Namen der Familien Ambiel, Amstalden, Bannwart und Wolf), um das Land, auf dem heute das historische Zentrum der Kolonie Helvetia steht, zu kaufen. Dort haben sie den bereits 1885 gegründeten Schützenverein Tiro ao Alvo neu angesiedelt, 1894 die Schweizer Schule São Nicolau de Flüe gegründet und 1889 die katholische Paróquia Nossa Senhora de Lourdes gebaut. Die zweite Gruppe von Obwaldner Einwanderern verschaffte sich auf diese Weise ein funktionierendes kulturelles Umfeld und konnte so das $U r$ Obwaldnertyytsch über lange Zeit bewahren.

\footnotetext{
${ }^{3}$ Aus Schweizer Sicht wollte man sich der ,unbrauchbare(n), verkommene(n) Individuen“ (DEWULF, 2007, S. 114) entledigen, während Brasilien das leere Land besiedeln und dabei neue europäische Arbeitskräfte nutzen wollten, um die Bevölkerung ,weißer“ und „reiner“ zu machen (u.a. OLIVEIRA, 2001).
} 


\section{$=$ TRAMA $=$}

\section{Der Sprachwechsel $^{4}$}

Verschiedene Faktoren haben dazu beigetragen, dass die Herkunftssprache von immer weniger Helvetianern $^{5}$ gesprochen wird. Zum Sprachenwechsel hat vor allem aber die Schulgeschichte im Zusammenhang mit den beiden Weltkriegen in Helvetia ihre Spuren hinterlassen:

Bis 1905 unterrichteten die Auswanderer ihre Kinder selbst, danach übernahmen bis 1917 die Katharinenschwestern aus Braunsberg/Deutschland den Schuldienst. Die Kommunikation innerhalb der Kolonie erfolgte zu Beginn, wie auch in vielen anderen deutschsprachigen Kolonien, in der mit einem hohen Prestige versehenen Herkunftssprache Ur-Obwaldnertyytsch. Die Sprachgemeinde war zwar nicht übermässig groß, dafür aber homogen; ökonomische Angelegenheiten konnten größtenteils innerhalb der weitgehend isolierten Sprachinsel geregelt werden. Bei auftretender Notwendigkeit wurden bilinguale Sprecher oder Übersetzer eingesetzt (WEIZINGER, 2005).

Während des Ersten Weltkriegs wurden zunächst alle deutschsprachigen Schulen geschlossen (u.a. Dekret Nr. 1.063, von 1917), aber die Schule São Nicolau de Flüe konnte dank des Einsatzes des Schulvorstandes in Helvetia schon bald unter einer Bedingung ihre Türen öffnen: Es mussten brasilianische Lehrkräfte eingestellt werden, die Portugiesisch, brasilianische Landes- und

\footnotetext{
${ }^{4}$ Im Gegensatz zum Dachsprachenwechsel (u.a. ALTENHOFEN, 2013), wo eine Standardsprache eine andere ablöst, kann mit Sprachwechsel auch eine Nicht-Standardsprache abgelöst werden, so wie es in der Kolonie Helvetia der Fall war.

${ }^{5}$ Helvetianer sind a) in Helvetia geborene Brasilien-Schweizer mit Schweizer Vorfahren, oder b) von a) akzeptierte Brasilianer, die sich kulturell (aber nicht sprachlich) der Gemeinde angepasst
}

Geschichtskunde unterrichten sollten. Damit begann der Prozess der Nationalisierung „fremder" Bürger (KREUTZ, 2000). Bis 1943 lehrten die Benediktinerinnen aus Sorocaba die Kinder der Brasilien-Schweizer. Deutsch, Schweizer Geographie und -Geschichte waren noch obligatorische Fächer an der Schweizer Schule auf brasilianischem Boden. $\mathrm{Zu}$ diesem Zeitpunkt vollzog sich der allmähliche Wechsel von der fast monolingualen Schweizerdeutschen Kolonie Helvetia zur externen Diglossie und Mehrsprachigkeit mit dem brasilianischen Portugiesisch (u.a. FERGUSON, 1971; KLOSS, 1966, 1986) ${ }^{6}$. Als Alltagssprache diente innerhalb der Kolonie nach wie vor Deutsch (bzw. das $U r$ Obwaldnertyytsch), jedoch wurde vor allem mit Personen ohne direkte Verbindung zur Kolonie nun notwendigerweise Portugiesisch gesprochen.

Während des Zweiten Weltkriegs wurden wiederrum alle ausländischen Schulen geschlossen (u.a. Dekret Nr. 88, von 1938), da Getúlio Vargas, damaliger Präsident des Estado Novo, - Adolf Hitler folgend - das Lema: „Ein Land, eine Sprache" umzusetzen versuchte. Die Schule São Nicolau de Flüe durfte zwar nach Kriegsende wiedereröffnet werden, jedoch ohne Deutschunterricht. Gleicherweise untersagte die neue Sprachpolitik des Landes das Sprechen von fremden Sprachen: Zeitschriften und Radiosendungen wurden unterbunden und in der Kirche durften Messen nur noch in portugiesischer Sprache abgehalten werden (GERTZ, 1991). Ab diesem Moment

haben. In diesem Artikel beziehen wir uns, wenn wir von Helvetianern sprechen, nur auf solche mit Schweizer Vorfahren.

${ }^{6}$ So wie auch in anderen deutschsprachigen Kolonien gab es zu Beginn der Einwanderung keinen stetigen Sprachkontakt zwischen der deutschen Varietät und dem Portugiesischen, da sich die meisten Siedlungen auf dem Land befanden ( $c f$. ROCHE, 1966; ALTENHOFEN, 2000). 


\section{$=$ TRAMA $=$}

kann von einer internen Diglossie ( $c f$. KLOSS, 1966, 1986) gesprochen werden, da das Ur-Obwaldnertyytsch nun zunehmend auch in den verschiedensten Bereichen innerhalb Helvetias von der neuen Prestigesprache Portugiesisch abgelöst wurde. Ein weiterer wichtiger Faktor für den Sprachwechsel waren die immer besser ausgebauten Transportmittel (Zug und Bus) und die moderner werdenden Medien (Internet, Radio, Telefon und TV). Obwohl es in Brasilien noch bis in die 1990er Jahre mehrere Hundert Sprecher des UrObwaldnertyytsch gab ( $c f$. AMSTALDEN, 1988), sind es heute nur noch 29 Helvetianer der älteren Generation, die den Urdialekt mehr oder weniger fließend sprechen.

Seit 2015 wird wieder aktiv an der außerschulischen Einführung des Deutschen in der Schweizer Kolonie gearbeitet. Große und kleine BrasilienSchweizer werden dabei in mehreren Kleingruppen von zwei Deutschlehrern unterrichtet. Der ursprüngliche Plan, Obwaldnertyytsch und Hochdeutsch gleichzeitig zu unterrichten, wurde jedoch schnell verworfen, da es für alle Interessierten unmöglich war, zwei deutsche Varietäten parallel zu erlernen. Zurzeit wird daher ausschließlich Hochdeutsch gelehrt, laut Angaben der Teilnehmenden aber mit mäßigem Erfolg. Vorerst ist somit in der Kolonie Helvetia noch nicht mit einem Revival der Herkunftssprache zu rechnen.

\section{V italitätsstatus und Sprachdomänen}

Um den Status von Minderheitensprachen in Hinlick auf die einzelnen Stufen des Spracherhalts zu klassifizieren, hat Fishman (1991) die Graded Intergenerational Disruption Scale (GIDS) erarbeitet. Im Jahr 2003 haben Brenzinger et al. im Auftrag der UNESCO diese Tabelle mit Hilfe der $E x$ panded Graded Intergenerational Disruption Scale (EGIDS), die mehr Kategorien gegen Ende des Sprachverlustprozesses enthält, erweitert. Ihre Typologie der Sprachvitalität geht von der Ebene 0 (lingua franca, international genutzte Sprache) bis hin zur Ebene 10b (language death, die Sprache wurde vergessen und wird weder gesprochen noch für kulturelle Zwecke angewandt).

Das Ur-Obwaldnertyytsch der Kolonie muss laut dem EGIDS klar hinter der Ebene 7 (shifting, d.h. Sprachwechselprozess, wo die Sprache zwar noch verstanden und gesprochen, aber den Nachfahren nicht mehr weitergegeben wird) eingeordnet werden, da die Brasilien-Schweizer bereits beinahe komplett akkulturiert ${ }^{7}$ und assimiliert ${ }^{8}$ sind. Der Sprachwechsel ist so gesehen vollzogen und der damit verbundene Prozess abgeschlossen. Der Dialekt Helvetias muss demnach zwischen Ebene 8a (moribunde Sprache, aktive Sprecher sind nur noch die Großeltern) und Ebene $8 \mathrm{~b}$ (fast ausgestorbene Sprache, nur noch die Großeltern oder ältere Menschen sprechen und nutzen diese Sprache) ein-

\footnotetext{
${ }^{8}$ In diesem soziokulturellen Prozess wird eine Minderheit nicht nur vom Hetero-Stereotyp geduldet und akzeptiert, sondern als bereichernd angesehen. Beispielsweise wird das kulturellen Erbe Helvetias sehr gepflegt: es wird gejodelt, Jass gespielt, zu Schweizer Volksmusik getanzt und es werden im Jahreskreis mehrere Schweizer Feste gefeiert, wo alle Interessierten herzlich willkommen sind.
} 


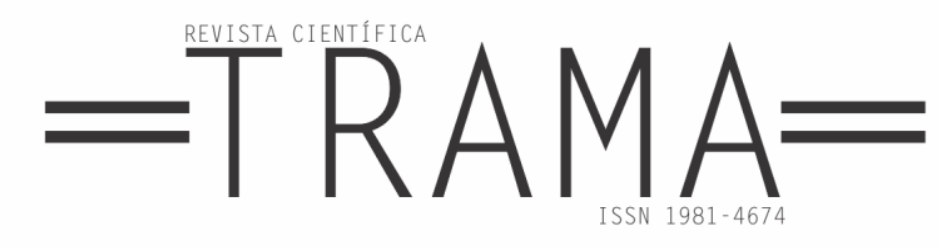

gestuft werden. Die UNESCO klassifiziert diese beiden Ebenen als "severely“ bzw. ,critically endangered languages“.

Das Ur-Obwaldnertyytsch ist daraus folgend eine vom Aussterben bedrohte Varietät. Stellt man in diesem Kontext die berühmte Frage von Fishman (1965) (Wer spricht welche Sprache wie, wann, mit wem...?), so behaupten viele Helvetianer:

(1) Poucas pessoas falam o dialeto.

(2) Só os velhos falam suíço.

(3) Não se tem com quem falar dialeto.

(4) Em casa ainda se consegue falar dialeto.

(5) Par que aprender o suiço? Estamos no Brasil! ${ }^{9}$

Die Informanten meiner ersten Feldforschung ${ }^{10}$ sind sich darin einig, dass nur noch ältere Zweisprachige den Dialekt beherrschen und diesen nicht mehr häufig anwenden:

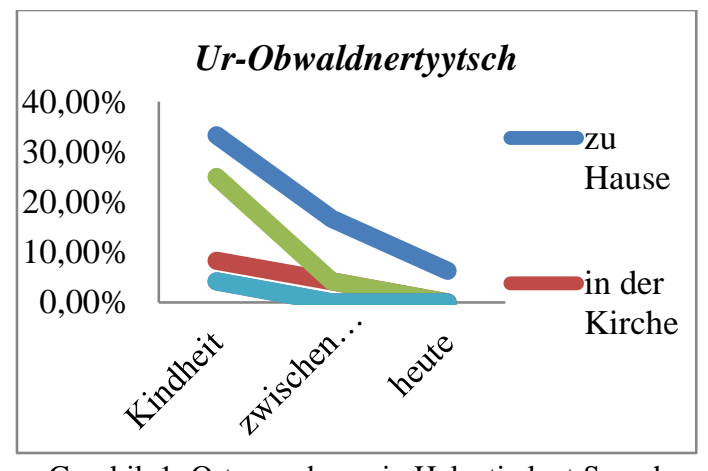

Graphik 1: Orte, an denen in Helvetia laut Sprecheraussagen noch Dialekt gebraucht wird

Geschwister oder Cousins nutzen manchmal Treffen, um im Dialekt über die Vergangenheit zu sprechen. Dies

9 Deutsche Übersetzung:

(1) Wenige Menschen sprechen den Dialekt.

(2) Nur die Alten sprechen Schweizerdeutsch.

(3) Man hat niemanden, mit dem man Dialekt sprechen kann.

(4) Zu Hause gelingt es einem noch Dialekt zu sprechen.

(5) Für was Schweizerdeutsch lernen? Wir sind in Brasilien!

geschieht meistens im familiären Umfeld. Auch sind bilinguale Helvetianer durchaus gewillt, ihr Können hinsichtlich ihrer Herkunftssprache mit Besuchern aus der Schweiz unter Beweis zu stellen:

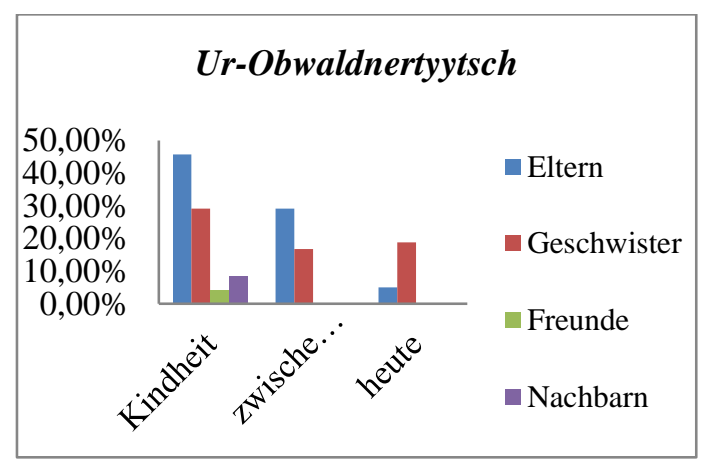

Graphik 2: Personen, mit denen in Helvetia laut Sprecheraussagen noch Dialekt gebraucht wird

Die weiter oben aufgeführte sprachpolitische Situation der Kolonie Helvetia deckt sich mit den hier aufgeführten Ergebnissen der ersten Feldforschung. Es wird immer weniger und in immer weniger Bereichen $U r$ Obwaldnertyytsch gesprochen.

Heutzutage fällt es den meisten Zweisprachigen schwer ein Gespräch ausschließlich auf Schweizerdeutsch zu führen. Oft kommen dabei unbewusst Interferenzen vor. Betroffen sind vor allem Bereiche der Phonetik, Morphologie, Syntax und Prosodie.

Auch bei den mittlerweile monolingual lusophonen Helvetianern sind einzelne Sprachbrocken des moribunden Ur-Obwaldnertyytsch zu erkennen. Diese wurden durch den langjährigen Spra-

\footnotetext{
${ }^{10}$ Die erste Feldforschung fand zwischen Juni und August 2015 statt. 32 Informanten (20 Männer und 12 Frauen aus 4 Generationsgruppen und 1 Gruppe Brasilianer ohne Schweizer Herkunft, jedoch mit regelmäßigem Kontakt zu Helvetia) haben einen soziolinguistischen Fragebogen ausgefüllt. Außerdem wurden spontansprachliche Äusserungen mittels Beobachtungen und Nacherzählungen festgehalten.
}

\section{.}




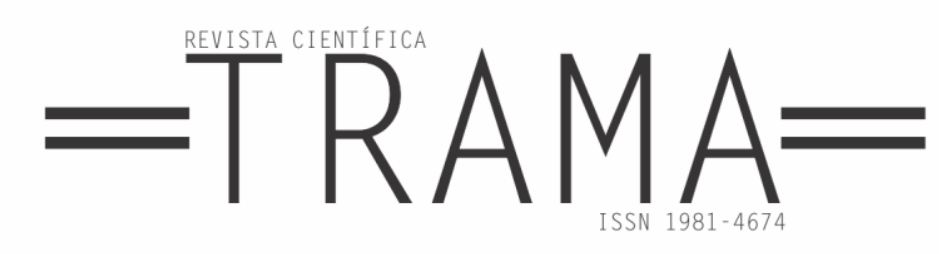

chkontakt in ihre Alltagssprache übertragen. Wörter wie „Hallo“, „Cheli ${ }^{11 ، “}$ und „Guätä Sunnti ${ }^{12 ، ~}$ sind gängige Ausdrücke, die jedoch mit einem starken portugiesischen Akzent wiedergegeben werden. Auch sind bei den jüngeren Helvetianern manchmal code-switchings und hybride Wortneubildungen $\mathrm{zu}$ erkennen, die innerhalb bestimmter Gruppen erschaffen und auch hauptsächlich innerhalb dieser Runde genutzt und verstanden werden.

\section{Sprachkontaktphänomäne}

Dass in einer Sprachgemeinde wie Helvetia Sprachkontaktphänomene auftauchen ist offensichtlich, vor allem wenn dort zwei Sprachen (beziehungsweise zwei Varietäten zweier Sprachen) über mehr als ein Jahrhundert koexistierten und sich damit gegenseitig beeinflussten.

In diesem Kapitel werden nun abschliessend einige konkrete Sprachbeispiele erläutert. Der Dialekt wird dabei sowohl aus der Sicht der bilingualenals auch aus der der monolingualen Helvetianer betrachtet.

\section{Bilinguale Sprecher}

Unter den zweisprachigen Sprechern müssen Abstufungen gemacht werden. Je besser ein Brasilien-Schweizer das Ur-Obwaldnertyytsch beherrscht bzw. je mehr er es im Alltag verwendet,

\footnotetext{
${ }^{11}$ Ursprünglich ist das „Cheli“ ein Kaffee mit Schnaps. Heute ist in Helvetia das Cheli ein Milchkaffee, mit mehr Milch als Kaffee und ohne Alkohol. Auch wurde ein Fußballclub nach dem Obwaldnergetränk benannt.

${ }^{12}$ Der wöchentliche Besuch der Kirche wird in Helvetia hoch angerechnet, da die meisten Helvetianer sehr katholisch sind. Nach der Messe wurde früher immer Dialekt gesprochen, gewisse Grußwörter wie „Guäte Sunnti“ haben dabei
}

desto weniger Sprachkontaktphänomene kommen zum Vorschein.

AMSTALDEN (1988) hat zum 100-jährigen Jubiläum der Kolonie ein Heft mit Auswertungen herausgegeben, die sie anhand von Fragebögen zusammengestellt hat. 4607 Helvetianer wurden befragt - davon 609 noch in der Kolonie lebend - welche die folgenden Angaben zum Sprachgebrauch machten:

\begin{tabular}{|l|l|}
\hline Sprechen Dialekt & $134(22 \%)$ \\
\hline $\begin{array}{l}\text { Sprechen ein wenig Dia- } \\
\text { lekt }\end{array}$ & $209(34.3 \%)$ \\
\hline Sprechen nicht Dialekt & $266(43.7 \%)$ \\
\hline gesamt & $609(100 \%)$ \\
\hline \multicolumn{2}{|c|}{ Tabelle 1: Dialektgebrauch der Helve- } \\
tianer im Jahr 1888
\end{tabular}

In meiner ersten Feldforschung habe ich 32 Informanten befragt, davon 24 Sprecher mit Schweizer Vorfahren. Diese machten folgende Angaben:

\begin{tabular}{|l|l|}
\hline $\begin{array}{l}\text { Sprechen Dialekt (fli- } \\
\text { eßend oder gut) }\end{array}$ & $5(21 \%)$ \\
\hline $\begin{array}{l}\text { Sprechen Dialekt (mäßig } \\
\text { oder ein wenig) }\end{array}$ & $12(50 \%)$ \\
\hline Sprechen nicht Dialekt & $7(29 \%)$ \\
\hline gesamt & $24(100 \%)$ \\
\hline
\end{tabular}

Tabelle 2: Dialektgebrauch der Helvetianer im Jahr 2015

Da es Sprecher gibt, die noch sehr gut Ur-Obwaldnertyytsch sprechen, und solche die es einfach noch verstehen und nur noch brockenweise wiedergeben können, werden im Folgenden nur Sprachbeispiele $^{13}$ von bilingualen Helvetianern gezeigt, welche den Dialekt noch gut beherrschen. Wir gehen an dieser

auch bei den Helvetianern überlebt, die kein $U r$ Obwaldnertyytsch sprechen.

${ }^{13}$ Da diese Sprachbeispiele in meinem Dissertationsprojekt noch genauer betrachtet werden, wird auf die Aufführung der Details der einzelnen Sprecher in diesem Artikel verzichtet ( $c f$. KARNOPP, in Arbeit). Es sind jedoch alles Informanten der ältesten beiden Sprachgruppen der Brasilien-Schweizer. 


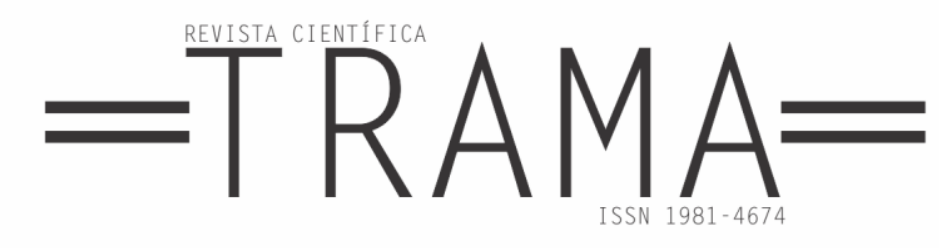

Stelle jedoch nicht von einem perfekten Bilingualismus laut BLOOMFIELD (1935) aus, sondern folgen eher der Definition von OKSAAR (1980, S. 43):

\begin{abstract}
Mehrsprachigkeit definiere ich funktional. Sie setzt voraus, dass der Mehrsprachige in den meisten Situationen ohne weiteres von der einen Sprache zur anderen umschalten kann, wenn es nötig ist. Das Verhältnis von Sprachen kann dabei durchaus verschieden sein - in der einen kann, je nach der Struktur des kommunikativen Aktes, u.a. Situationen und Themen, ein wenig eloquenter Kode, in der anderen ein mehr eloquenter verwendet werden.
\end{abstract}

Code-Switchings kommen bei diesen Zweisprachigen sowohl interphra$\mathrm{sal}^{14}$ als auch intrasphrasal ${ }^{15}$ vor. Beispielsweise in folgendem Aus-schnitt einer Bildergeschichtserzählung:

\begin{abstract}
Aso dahie isch ä Famili wo wohnt ufm Land und het es schens Huis und es schens Vorlaibeli mit $\ddot{a}$ schenä Sässel. Und hend Hiändli und hend Chiä und hend hie so Färli und hend es Windrod. Und dahie sind si am Land ussä em Huis vorussä, und dFroi isch am dHiändli hiete, und $d r M a$ isch am Holz spaltä, und sGoof sBiebli isch am futebol spile. Outra coisa que vocês falam: DGoofä tiend bringgä.
\end{abstract}

\footnotetext{
14 Bezeichnet den Austausch von sprachlichem Material zwischen isolierten Sätzen oder Satzteilen.

${ }^{15}$ Diese Satzteile oder Wörter können an einer beliebigen Stelle im Satz auftauchen. Das Mixing kommt meistens dann vor, wenn beide Sprachen ziemlich gut beherrscht werden.

${ }^{16}$ Laut HOFFMANN (1991) gibt es verschiedene Auslöser, weshalb ein Sprecher ein Wort der anderen Sprache in den Satz einbaut: a) wenn er das Wort in der Erzählsprache nicht kennt; b)
}

Interphrasal ist hier die Aussage „Outra coisa que vocês falam“, da die Informantin ohne Vorwarnung und unbewußt auf Portugiesisch spricht und dann, im nächsten Satz, gleich wieder zu Ur-Obwaldnertyytsch wechselt. Kommen Sprecher also von einer Erzähllinie $a b$, ist der Wechsel in die Alltagssprache nicht ungewöhnlich, wie auch im folgenden Beispiel ersichtlich ist:

$$
\begin{aligned}
& \text { Wie seit mr lanche'? Não sei. } \\
& \text { Como se diz lanche? Odr 'san- } \\
& \begin{array}{l}
\text { duiche', mas isto também é por- } \\
\text { tuguês. }
\end{array}
\end{aligned}
$$

Als intraphrasales Beispiel in (6) benutzt die Informantin das portugiesische Wort futebol anstatt dem Schweizerdeutschen Fuessball, wahrscheinlich, weil sie letzteres nicht kennt und weil das Wort in der Mehrheitssprache zweifellos gebräuchlicher ist ${ }^{16}$.

Der Begriff „bringgä“ (spielen) existiert weder im Portugiesischen noch im Schweizerdeutschen und ist ein klares Beispiel für eine hybride Neubildung ${ }^{17}$. Hier wurden die Wörter brincar und das Schweizerdeutsche Suffix - $\ddot{a}^{18}$ zusammengefügt. Ein weiteres sehr oft benutztes Verb in Helvetia ist ",tuncar", abgeleitet vom Schweizerdeutschen tunkä oder tünklä (nass machen) in Kombination mit dem Portugiesischen Suffix der Verbinfinitivform -ar. Auch werden oft Hybridismen mit Substantiven gebildet: wenn ein Begriff temporär nicht zur Verfügung steht; c) wenn ein Begriff nur sehr wenig in der anderen Sprache gebraucht wird, und; d) wenn ein Kind die Inputs verwechselt.

${ }^{17}$ Ein bilingualer Sprecher organisiert seine Rede in der Sprache A, aber fügt Morpheme der Sprache B ein, sodass Neologismen entstehen können.

${ }^{18}$ Das Suffix - $\ddot{a}$ signalisiert bei Schweizerdeutschen Verben den Infinitiv: machä (machen), spilä (spielen), essä (essen). 


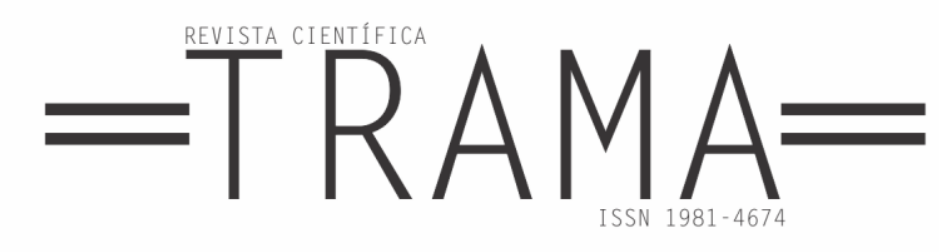

DFroi tiot Mili dr Hiändli gä.

In diesem Beispiel wird anstatt dem (schweizer)deutschen Wort Mais, das portugiesische milho verdeutscht.

\section{Was tiot si mache? En} Sandwisch.

Das Sandwich stammt klar aus der englischen Sprache, und wird heute genauso im schweizerdeutschen Dialekt gebraucht. Der Begriff beleiti Bröötli ist seltener. Dennoch scheint im Beispiel (9) eher eine Interferenz vom portugiesischen sanduíche stattgefunden zu haben.

Eine syntaktische Interferenz finden wir im Sprachbeispiel (6) im Relativsatz ,wo wohnt ufem Land“ (que mora no сатро). Heute ist es jedoch üblicher zu sagen: „wo ufem Land wohnt“. Anstelle der Verbendstellung im Nebensatz finden wir dort die portugiesische Satzstellung. Solche Interferenzen sind bei den mehrsprachigen Brasilien-Schweizern häufiger und finden sich auch in folgenden Beispielen wieder:

Wo hend oi Händeli und Veh (que tem também galinhas e gado) vs. Wo oi Hiändeli und Veh hend.

D Froi wo isch $i$ de Chuchi (a mulher que está na cozinha) vs. $D$ Froi wo i de Chuchi isch.

$\mathrm{Da}$ es im Portugiesischen nur eine Präposition para für die deutschen "für+Akkusativ" und "zu+Dativ" gibt, ist es für beinahe alle bilingualen Helvetianer schwierig, diese grammatikalisch korrekt anzuwenden:

(12) Für sii mitenand ässe vs. Zum mitenand ässe.

(13) Holz firs zässe mache vs. Holz zum zässe mache.

Alle hier aufgelisteten Sprachphänomene treten laut vergleichbaren Ergebnissen von ALTENHOFEN (1996), ROSENBERG (2003) und weiteren Sprachforschern dieses Gebiets ebenso häufig in anderen deutschsprachigen Kolonien Brasiliens auf.

\section{Monolinguale Sprecher}

Die einsprachigen BrasilienSchweizer sind ebenso wie die zweisprachigen sehr kreativ veranlagt, was ihre Sprache anbelangt, da diese durch ihre Eltern und/oder Großeltern lange Zeit in Kontakt mit dem Schweizerdeutschen Dialekt sind/waren.

Auffällige Sprachbeispiele finden sich vor allem in geschlossenen Kleingruppen wieder. Beispielsweise hört man bei einer Gruppe von Schwestern:

\section{(14) Isch mane hore.}

Dieser Satz stellt einen typischen Code-Mix zwischen dem Satz "isch mal a dr Zyt" und dem portugiesischen "está na hora" dar.

In der Volkstanzgruppe Helvetias können einige Helvetianer ein wenig Deutsch. Das Adjektiv schen (schön) ist weit verbreitet und wird auch oft benutzt. In der Tanzgruppe, als einst eine Aufführung äußerst gelungen war, wurde von einem Teilnehmer ein Neologismus gegründet:

\section{Xeníssimo.}

Das deutsche Superlativ 'sehr schön' ist teilweise bekannt, und dennoch haben sie mit dem Suffix des portugiesischen Superlativs -issimo ein eigenes und neues Wort kreiert. Viele zweisprachige Helvetianer verstehen dieses, wenden es jedoch nur selten an.

Eine Gruppe Cousinen der vierten Generation Brasilien-Schweizer hat dem Possessivpronomen vosso eine neue semantische Bedeutung gegeben. Grund dafür war ein japanischer Freund des Vaters einer jungen Helvetianerin, der sie fragte: „O vosso pai está em casa?“. Sie und ihre Cousinen fanden es witzig, dass dieser Mann noch immer die altmodische Anredeform vosso und nicht eine neuere wie seu oder de você benutzte. $\mathrm{Ab}$ 


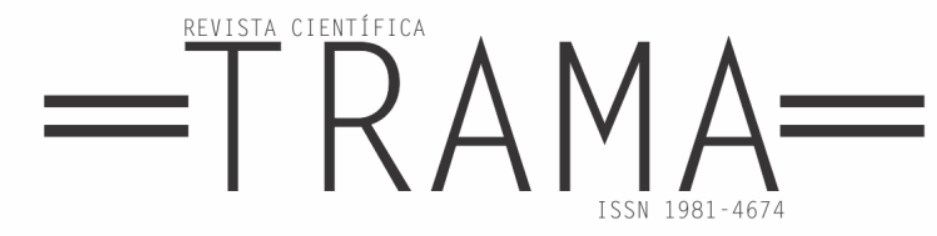

diesem Moment ist eine Person, die nicht der Norm entspricht, sich so benimmt oder anders gekleidet ist um vosso oder uma vossa.

\section{Schlussbemerkungen}

Da die Helvetianer schon weit fortgeschritten akkulturiert und assimiliert sind, ist von der lang andauernden aktiven Sprachkontaktphase nicht mehr viel Sprachmaterial vorzufinden. Dennoch gibt es bei genauem Betrachten verglichen mit anderen Sprachgruppen aus der Region São Paulo die eine oder andere Abweichung im Sprachgebrauch. Und genau diese Details bewirken, dass man sich in der Kolonie Helvetia, v.a. wenn man untereinander ist, aber auch im allgemeinen Sprachgebrauch, aufgrund sprachlicher Merkmale als Helvetianer identifiziert.

\section{B $_{\text {ibliografie }}$}

ALTENHOFEN, Cléo Vilson. Hunsrückisch in Rio Grande do Sul. Ein Beitrag zur Beschreibung einer Deutschbrasilianischen Dialektvarietät im Kontakt mit dem Portugiesischen. Stuttgart: Franz Steiner Verlag, 1996.

O português em contato com as línguas de imigrantes no Sul do Brasil. In: GÄRTNER, Eberhard; HUNDT, Christine; SCHÖNBERGER, Axel (Hg.). Estudos de geolingüística do português americano. Frankfurt am Main: TFM, 2000, S. 79-93.

Standard und Substandard bei den Hunsrückern in Brasilien: Variation und Dachsprachenwechsel des Deutschen im Kontakt mit dem Portugiesischen. In: LENZ, Alexandra N. (Hg.). German Abroad. Perspektiven der Variationslinguistik, Sprachkontakt- und Mehrsprachigkeitsforschung. Göttingen: Vienna University Press, 2016, S. 103-130.
AMSTALDEN, Leonor (ed.). A memória histórica de uma comunidade revela a identidade de sua gente. São Paulo: Imprensa oficial do Estado S.A. IMESP, 1988. BLOOMFIELD, Leonard. Linguistic Aspects of Science. In: Philosophy of Science, 2/4, 1935, S. 499-517.

BRENZINGER, Matthias; DWYER, Arienne M.; GRAAF, Tjeerd de; GRINEVALD, Colette; KRAUSS, Michael; MIYAOKA, Osahito; OSTLER, Nicholas; SAKIYAMA, Osamu; VILLALÓN, María E.; YAMAMOTO, Akira Y.; ZEPEDA, Ofelia. Language vitality and endangerment. Paris: UNESCO Ad Hoc Expert Group Meeting on Endangered Languages, 2003.

DEWULF, Jeroen. Brasilien mit Brüchen. Schweizer unter dem Kreuz des Südens. Zürich: Verlag Neue Zürcher Zeitung, 2007. FERGUSON, Charles A. Diglossia. In: DIL, Anwar S (Hrsg.) Language Structure and Language Use. Eassays by Charles A. Ferguson. Standford, CA.: Standford University Press, 1971 [1959], S. 1-26.

FISHMAN, Joshua. Yiddish in America: socio-linguistic description and analysis. Bloomington: Mouton, 1965.

Reversing language shift

Clevedon, UK: Multilingual matters Ltd, 1991.

GERTZ, René. O perigo alemão. Porto Alegre: Ed. Da Universidade, UFRGS; 1991.

HOFFMANN, Charlotte. An introduction to bilingualism. England: Longman, 1991.

Historisches Lekikon der Schweiz, Kartoffel. Einsehbar unter: http://www.hls-dhsdss.ch/textes/d/D13858.php. Zugang: 31.08.2017.

IMFELD, Karl. Obwaldner MundartWörterbuch. Kriens: Brunner, 2001.

KARNOPP, Andreia Caroline. Doktorarbeit am Romanischen Seminar der Universität Zürich zum Thema $\mathbf{O}$ português levemente diferente dos helvetianos em São Paulo, in Arbeit.

KLOSS, Heinz. Types of Multilingual Communities: A Discussion of Ten Variables. In: Sociological Inquiry. Volume 36, Issue 2, April 1966, S. 135-145.

. Über Diglossie. In: Zeitschrift

für Dialektologie und Linguistik. Wiesbaden, n. 5, 1986, S. 65-75.KREUTZ, Lúcio. 


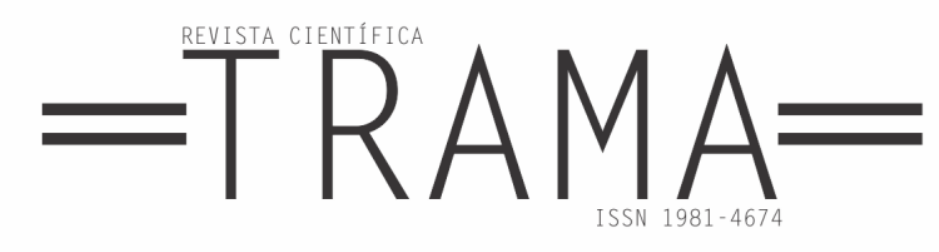

Escolas comunitárias de imigrantes no Brasil: instâncias de coordenação e estruturas de apoio. In: Revista Brasileira de Educação (Hrsg.). São Leopoldo: UNISINOS, 2000, S. 159-176.

OLIVEIRA, Lucia L. O Brasil dos imigrantes. Rio de Janeiro: Jorge Zahar Ed., 2001.

OKSAAR, Els. Mehrsprachigkeit, Sprachkontakt, Sprachkonflikt. In: NELDE, Peter H. (Hrsg.): Sprachkontakt und Sprachkonflikt. Wiesbaden: Steiner, 1980, S. 43-52.

ROCHE, Jean. As bases físicas da ocupação do solo no Rio Grande do Sul. In: AB'SÁBER, Aziz Nacib \& ROCHE, Jean (Hrsg.). Três estudos rio-grandenses. Porto Alegre: Faculdade de Filosofia da Universidade Federal do Rio Grande do Sul, 1966, S. 29-64. ROSENBERG, Peter. Vergleichende Sprachinselforschung: Sprachwandel in deutschen Sprachinseln in Russland und Brasilien. In: Linguistik Online, Bd. 13, Nr. 1, 2003, S. 273-324.

STREIFF-BECKER, Rudolf. Die Schweizerkolonien in Brasilien. Zürich: StreiffBecker, 1957.

WEIZINGER, Franz X. Die Schweizer Kolonie Helvetia in Brasilien: [18541935]. A colônia suíça Helvetia no Brasil. Tradução e adaptação por Maria Alvina Krähenbühl e Lydia Ming. Campinas: Sociedade de Tiro-ao-Alvo Helvetia, (2005 [1935]). 